\title{
Green tea polyphenol microparticles based on the oxidative coupling of EGCG inhibit amyloid aggregation/cytotoxicity and serve as a platform for drug delivery
}

Luiza Fernandes ${ }^{1}$, Beatriz Messias ${ }^{1}$, Antonio Pereira-Neves ${ }^{2}$, Estefania P. Azevedo ${ }^{1}$, Júlia Araújo $^{1}$, Debora Foguel ${ }^{1}$ and Fernando L. Palhano ${ }^{1 *}$

${ }^{1}$ Instituto de Bioquímica Médica Leopoldo de Meis, Programa de Biologia Estrutural, Universidade Federal do Rio de Janeiro, Rio de Janeiro, RJ, 21941-590, Brazil.

${ }^{2}$ Fiocruz Pernambuco, Instituto Aggeu Magalhães, Departamento de Microbiologia, Recife, PE, 50740-465, Brazil.

* To whom correspondence should be addressed: Fernando L. Palhano: Tel.: +55 21 3938-6761

E-mail: palhano@,bioqmed.ufrj.br

Pages S1-S7;

Figures S1-S6 


\section{Supplemental Figures}

A<smiles>O=C(O[C@H]1Cc2c(O)cc(O)cc2O[C@H]1c1cc(O)c(O)c(O)c1)c1cc(O)c(O)c(O)c1</smiles>

B

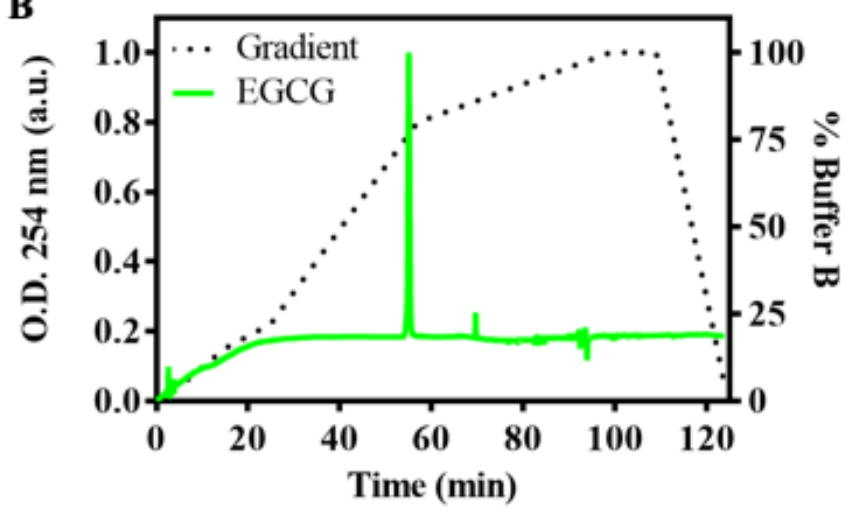

C

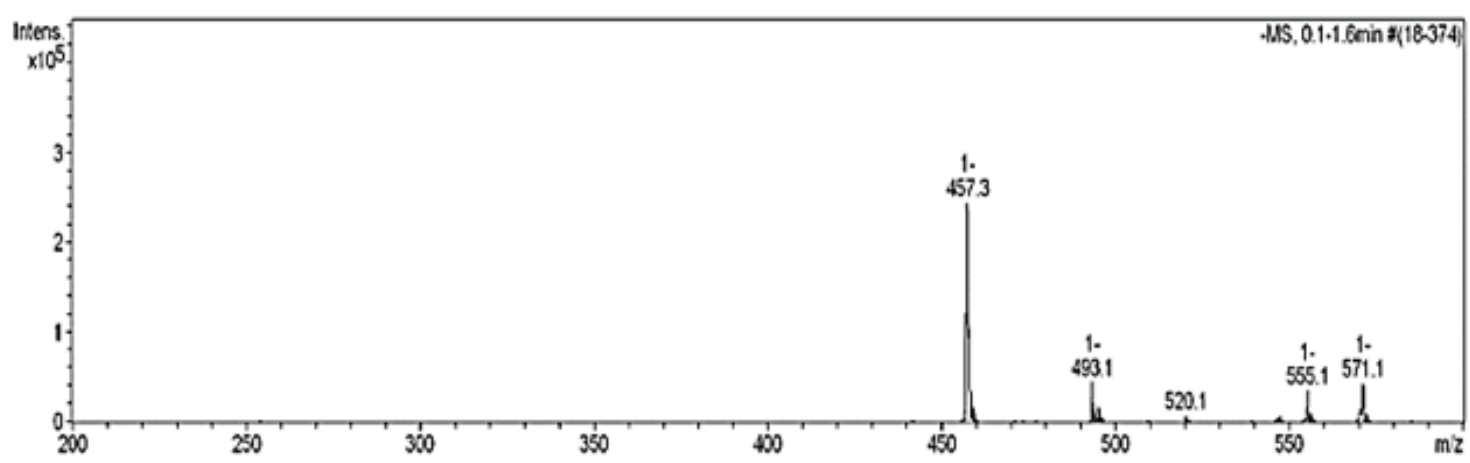

Figure S1. Characterization of epigallocatechin-3-gallate (EGCG). (A) Chemical structure of EGCG. (B) Analysis of EGCG by reversed-phase chromatography. Elution was monitored by the absorbance at $\lambda=254 \mathrm{~nm}$. (C) Electrospray ionization mass spectrometry was performed to measure the EGCG purity. 


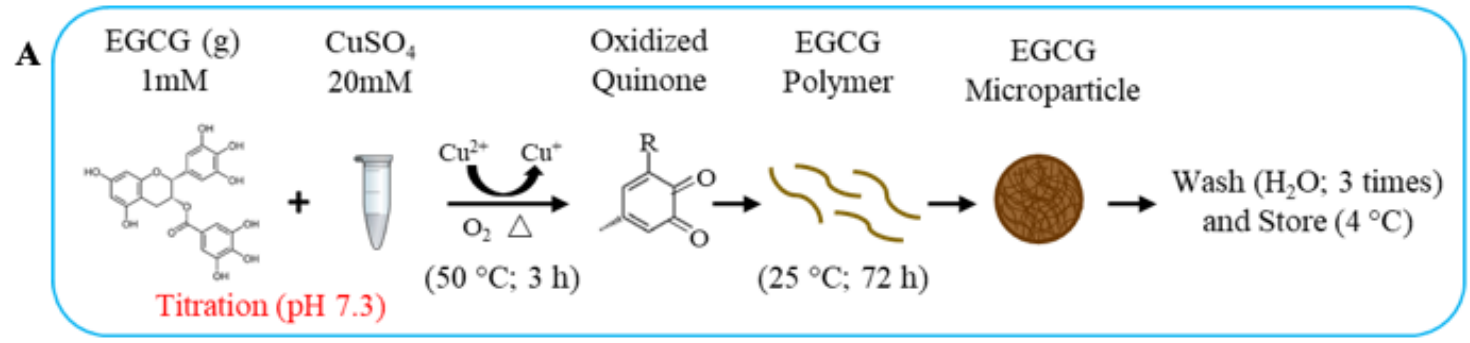

B
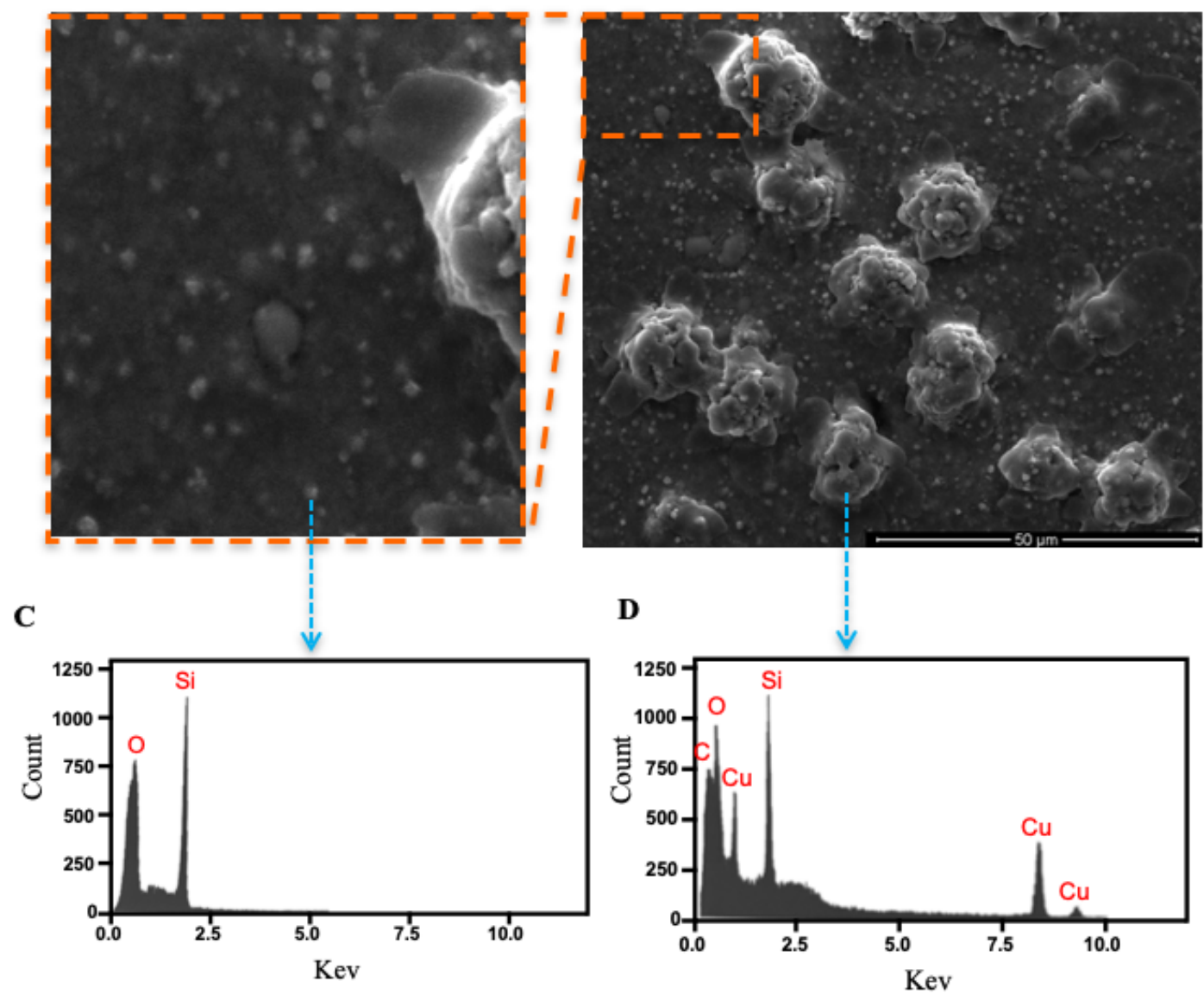

Figure S2. Scheme of the synthesis and characterization of EGCG microparticles (EGCG MPs). (A) Synthesis of EGCG MPs. EGCG (1 mM) was incubated with $\mathrm{CuSO}_{4}(20 \mathrm{mM})$, and the $\mathrm{pH}$ was titrated to 7.3. After $3 \mathrm{~h}$ at $50{ }^{\circ} \mathrm{C}$, polymers were formed, and during 3 days at $25^{\circ} \mathrm{C}$, spherical particles precipitated. (B) Analysis of EGCG MP morphology by high-resolution scanning electron microscopy. The orange dotted line represents a zoomed in view, and the blue arrows indicate two types of microparticles. The presence of copper $(\mathrm{Cu})$ was evaluated by energy dispersion X-ray microanalysis in the (C) small and (D) large microparticles. After observing that this protocol leads to copper precipitation, the EGCG MPs were always formed at pH 3.5. 

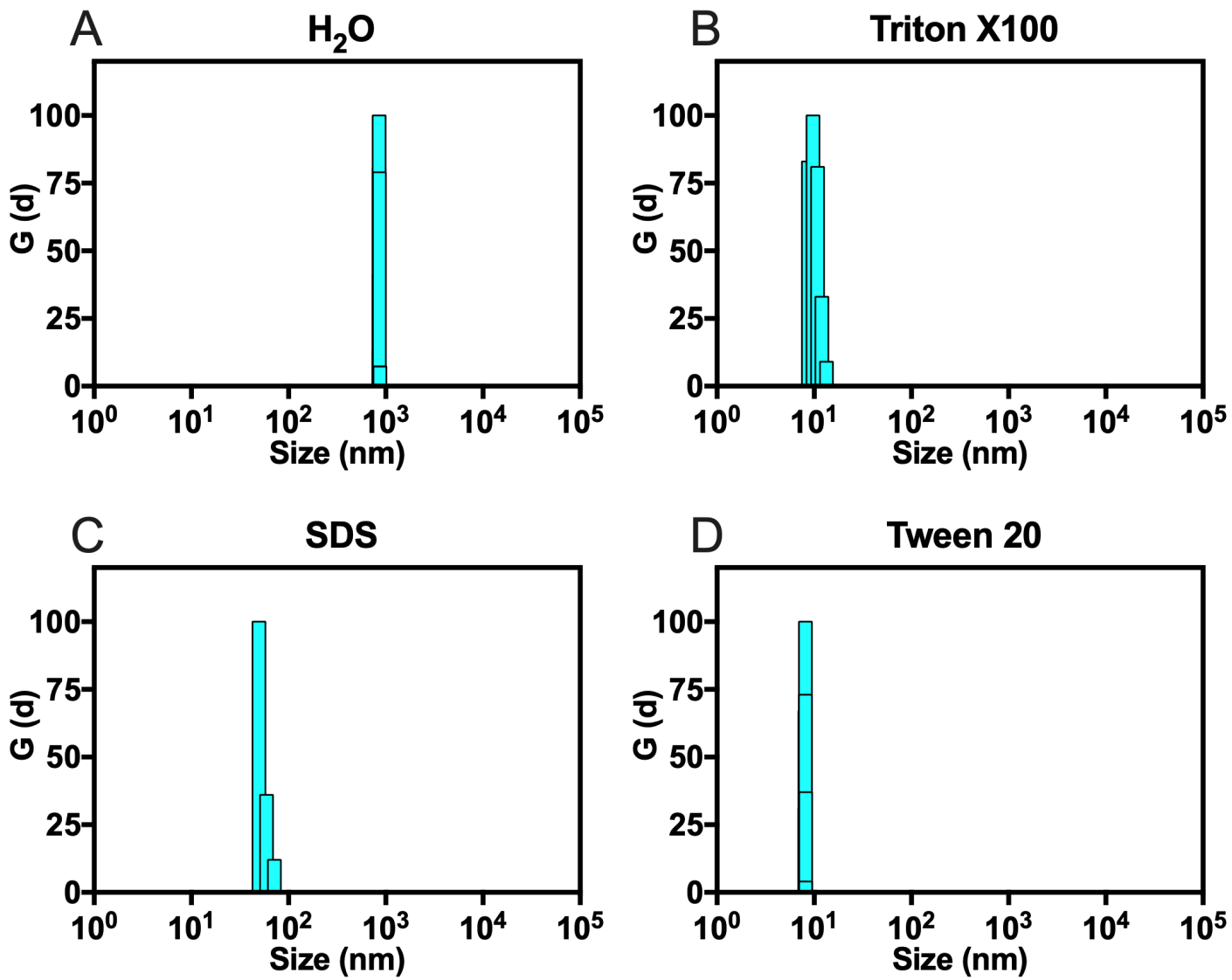

Figure S3. Dynamic light scattering analysis of the MPs. The MPs $(10 \mu \mathrm{M})$ were incubated at $25^{\circ} \mathrm{C}$ for 15 min with $100 \mathrm{mM}$ of Triton X100 (B), SDS (C), Tween 20 (D), NaCl or urea. As a control, MPs were incubated just with the solvent (A). The spectra of MPs incubated with $\mathrm{NaCl}$ or urea is identical of the control (A). 


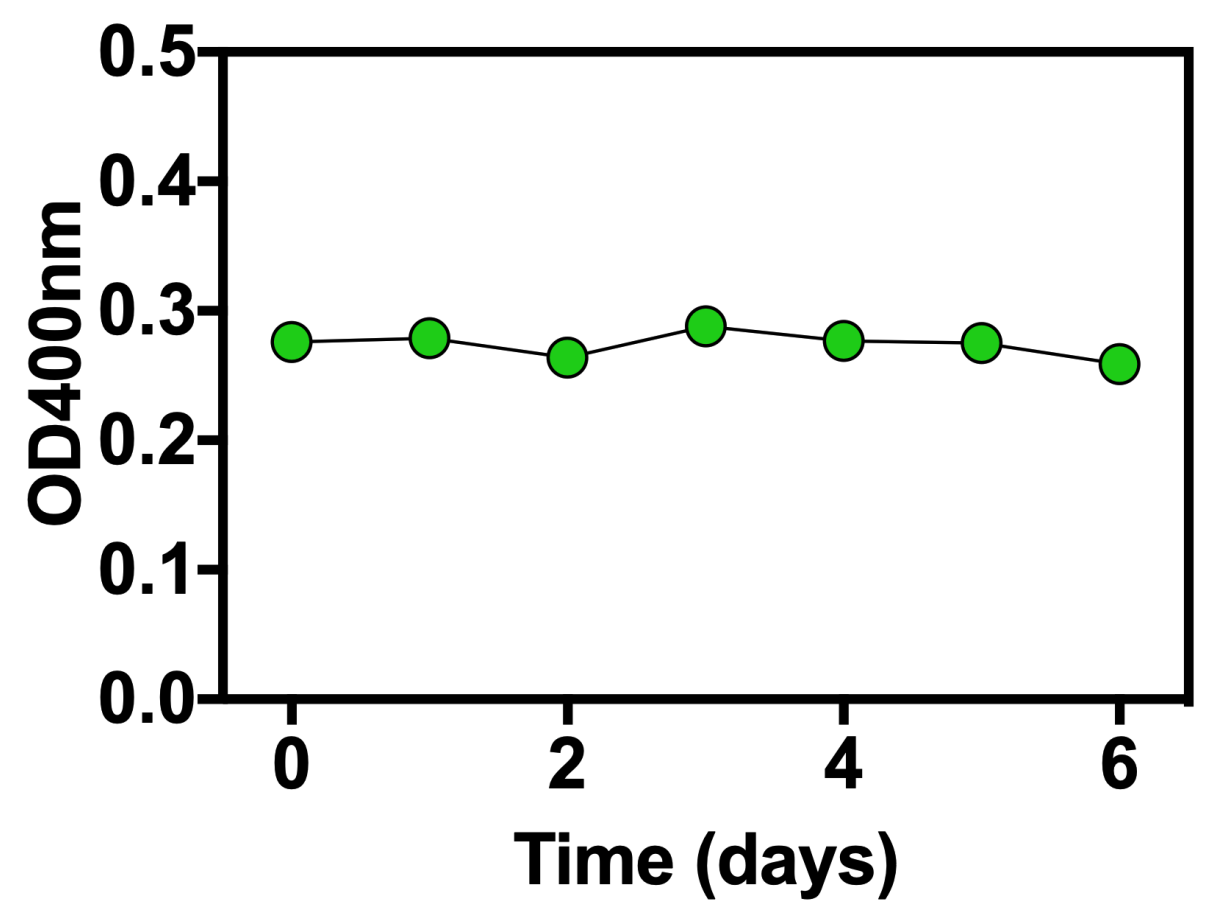

Figure S4. Stability of MPs over time. The MPs $(250 \mu \mathrm{M})$ were incubated at $25^{\circ} \mathrm{C}$ for 6 days and the turbidity was measured by optical density at $400 \mathrm{~nm}$ according with time. 


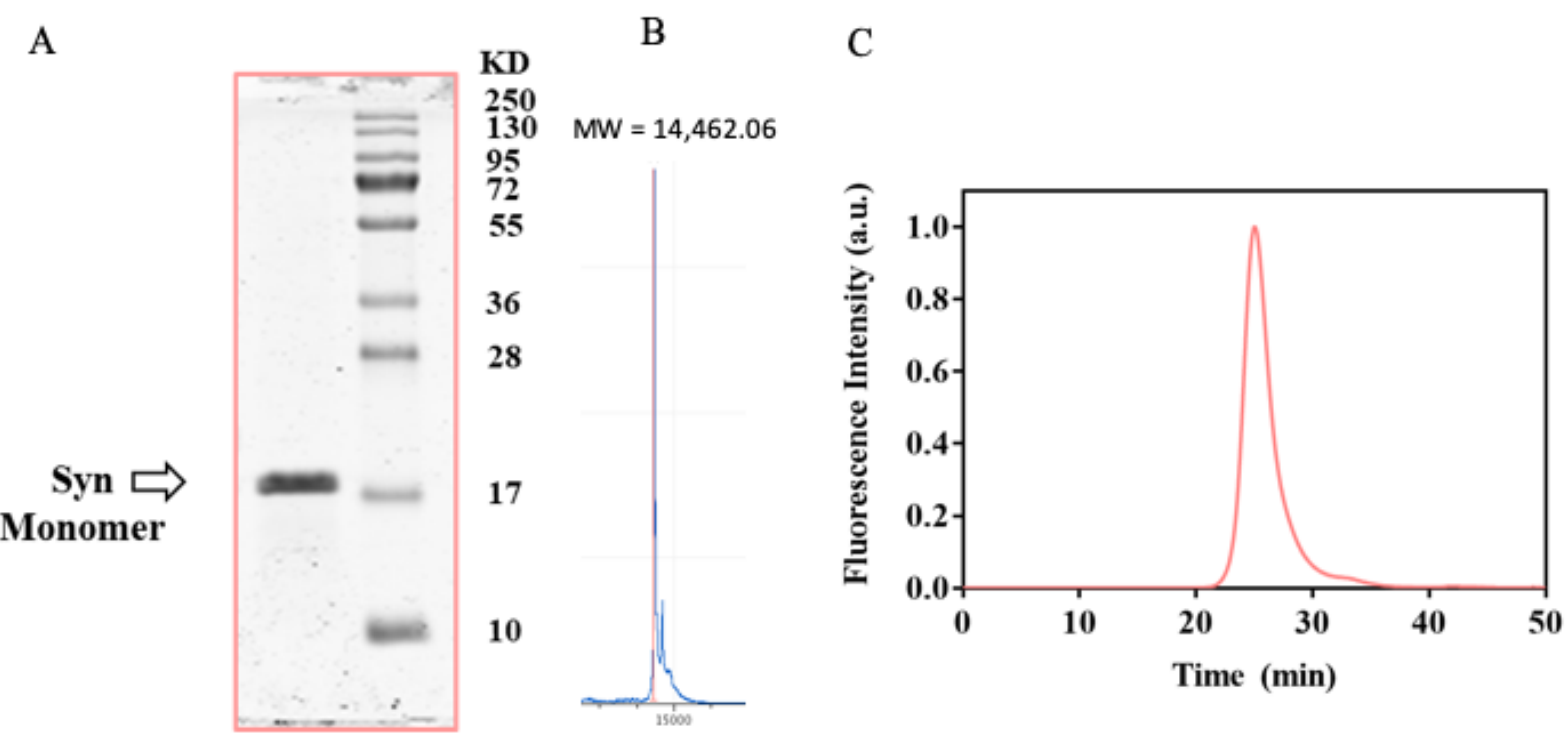

Figure S5. Characterization of monomeric alpha-synuclein ( $\alpha$-syn). (A) Electrophoretic mobility of monomeric $\alpha$-syn determined by $15 \%$ SDS-PAGE. (B) MALDI-TOF analysis of purified $\alpha$-syn showing a molecular weight of $14,462 \mathrm{kDa}$ (expected 14,460$)$. (C)

Chromatogram of monomeric $\alpha$-syn analyzed using a size-exclusion column (Superdex 75). 
A

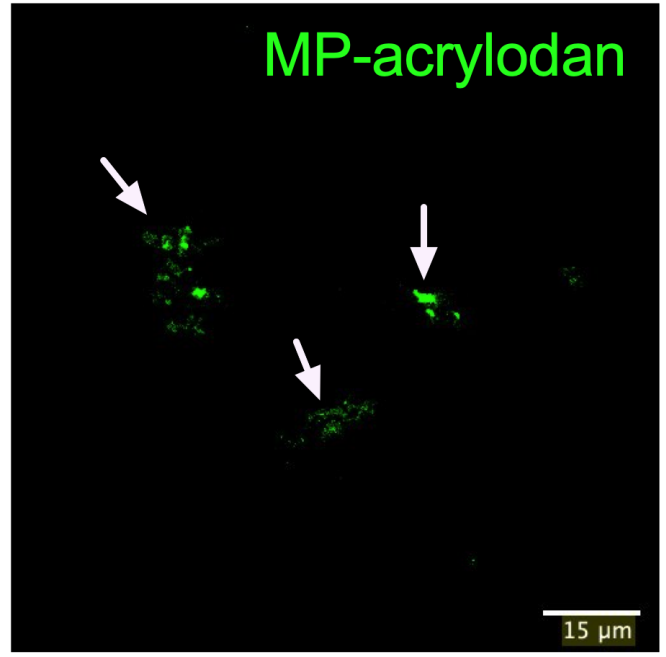

C

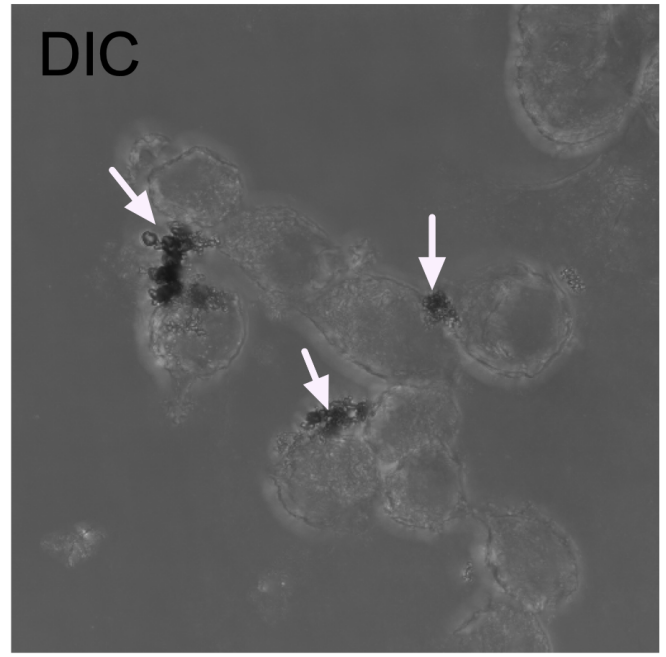

B

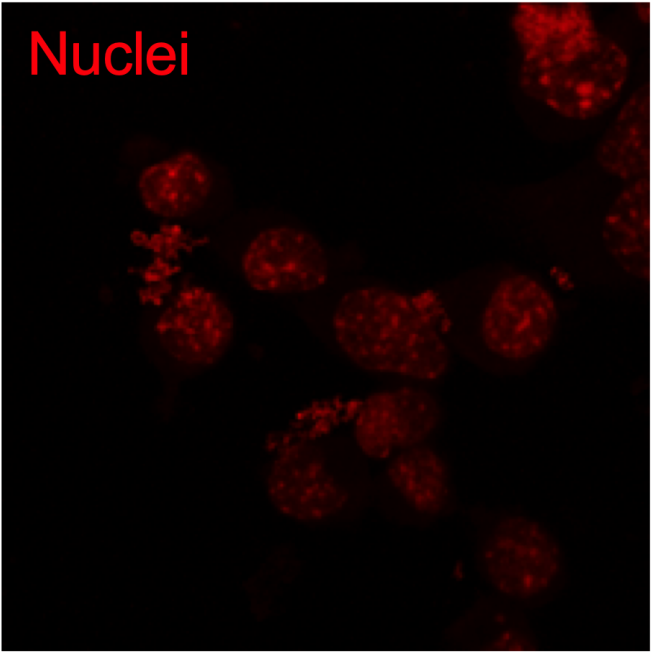

D

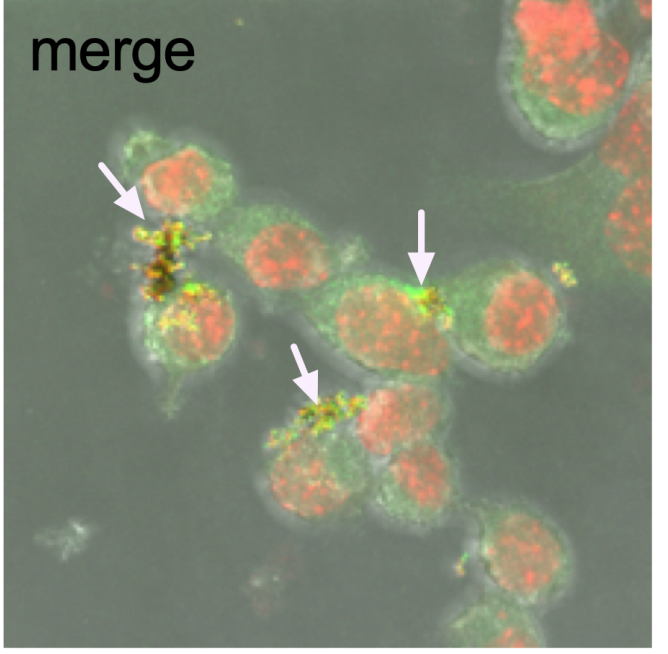

Figure S6 Confocal images of neuroblastoma cells treated with MPs. Fluorescence

microscopy was used to analyze the cellular capability to internalize MPs (A, green points)

loaded with the fluorescente probe acrylodan. The nuclei was stained with TO-PRO-3 (B, red). The arrows indicate the MPs in the surface of the cells. The cells were incubated with MPs (10 $\mu \mathrm{M})$ for $1 \mathrm{~h}$. 